\title{
The Pressure Effect on Glass Formation and Cluster Structure Evolution during Cooling Process of PdNi Alloy: A Molecular Dynamics Study
}

\author{
S. KAZANC ${ }^{a, *}$ AND F.A. CELIK $^{b}$ \\ ${ }^{a}$ Firat University, Faculty of Education, Physics Teaching Programme, 23119 Elazig, Turkey \\ ${ }^{b}$ Bitlis Eren University, Faculty of Arts and Sciences, Physics Department, 13000 Bitlis, Turkey
}

(Received October 16, 2012; in final form October 8, 2013)

Based on the embedded atom method a molecular dynamics simulation technique has been used to study the glass formation and atomic short range order in $\mathrm{Pd}-\mathrm{Ni}$ binary alloy. By using radial distribution functions and Honeycutt-Andersen pair analysis methods, the structural development of this alloy is studied by quenching from the liquid at two different cooling rates and under 0 and $5 \mathrm{GPa}$ pressures. The results show that the variation of local atomic bonded pairs is of great importance to understand the glass formation process and increase of the number of the ideal icosahedra under high pressure contributes to more ideal glass formation of model alloy.

DOI: $10.12693 /$ APhysPolA.125.49

PACS 64.70.pe, 83.10.Rs, 34.20.Cf

\section{Introduction}

Metallic glasses are fascinating engineering materials because of their mechanical and magnetic properties. The difficulty of observing a glass transition was the very high cooling rates up to $10^{6} \mathrm{~K} / \mathrm{s}$. To avoid the nucleation and growth of crystalline phase, one of the typical methods is to keep a high cooling rate in a range of $10^{6}-10^{12} \mathrm{~K} / \mathrm{s}$. However, due to the demand of a high cooling rate the rapid solidification method is restricted in most experimental case [1-5].

The $\mathrm{Pd}-\mathrm{Ni}$ based metallic glasses are of considerable scientific interest because they form bulk metallic glasses. Our interest in this material is related to its ability to form a basis for development of some industrially important bulk metallic glasses $(\mathrm{Pd}-\mathrm{Ni}-\mathrm{P}$ and $\mathrm{Pd}-\mathrm{Ni}-\mathrm{Cu}-\mathrm{P})$. Hence, the study of the properties of undercooled (amorphous) $\mathrm{Pd}-\mathrm{Ni}$ alloys is important for designing new bulk metallic glasses having technological importance [6].

The measurement of how an atom moves in a glass is not experimentally accessible. It is desirable to understand the origin of glass formation at the atomic level. This can be achieved by molecular dynamics (MD) simulation. Recently, research investigated the pressure effect on rapid solidification structure from liquid phase by using molecular dynamics method [7-10].

Atomic simulation techniques have become a powerful tool as they provide a physical insight in understanding various phenomena on atomic scale. MD simulations are used extensively to investigate the structural or thermodynamic properties of high technological materials such as intermetallic alloys, semiconductors, polymers or liq-

*corresponding author; e-mail: skazanc@firat.edu.tr uids in atomic scale $[11,12]$. Therefore, MD simulation is an excellent tool to study amorphous metal systems because of the dynamic processes associated with amorphous phase transformation and subsequent behavior. The MD technique consists of the numerical solution of the equations of motion for a system of $N$ atoms [13, 14]. The consistency of the results obtained from MD simulations with the experimental values depends tightly on the potential energy functions which are used to model the relevant system. On the other hand, the determination of the potential parameters, especially for the cross potential functions in alloy systems, is still a problem [11, 12]. One of the most useful potential energy methods is the embedded atom method (EAM) based on the many-body interactions proposed by Daw and Hatcher [15]. However, due to the simplicity of their potential functions, the versions of the EAM proposed by Vother-Chen [16], Finnis-Sinclair [17] and Sutton-Chen [18] are widely used to investigate metallic systems and its alloys [19, 20]. Although the first principles methods are the most rigorous, they are limited to very small systems and need the computers with higher speed [21].

In the present work, the MD method proposed by Parrinello and Rahman for the NPT ensemble is used to determine the pressure effect on the rapid solidification of liquid PdNi binary alloy system. To model the interactions between atoms Sutton-Chen type of embedded atom method based on many-body interaction was used. The liquid phase of PdNi alloy has been produced and solidified by applying the rapid quenching techniques under different pressure values. The structural properties are analyzed by means of radial distribution function, pair analysis as a function of temperature at various cooling rates and effect of the pressure to see whether the model alloy system goes into glassy structure. Glass state transition temperatures are predicted from the WendtAbraham parameter. 


\section{The method of calculations}

\subsection{Potential energy function}

In the present study a SCEAM potential function is used. The total energy of a crystal with $N$ atoms in the SCEAM methodology is given by [18, 22];

$$
E_{\mathrm{T}}=\sum_{i} E_{i}=\sum_{i}\left[\frac{1}{2} \sum_{j \neq i} \varepsilon_{i j} \phi\left(r_{i j}\right)-c_{i} \varepsilon_{i i} \sqrt{\rho_{i}}\right],
$$

where $r_{i j}$ is the separation between atom $i$ and $j, c$ is a positive dimensionless parameter, $\varepsilon$ is a parameter with the dimensions of energy. $\phi\left(r_{i j}\right)$ is a pairwise repulsive potential

$$
\phi\left(r_{i j}\right)=\left(\frac{A_{i j}}{r_{i j}}\right)^{n}
$$

between atoms $i$ and $j$ (arising primarily from the Pauli repulsion between the core electrons), while the metallic bonding is captured in a local energy density, $\rho_{i}$, associated with the atom $i$ and defined as

$$
\rho_{i}=\sum_{j \neq i} \rho\left(r_{i j}\right)=\sum_{j \neq i}\left(\frac{A_{i j}}{r_{i j}}\right)^{m} .
$$

Here $A$ is a parameter with the dimensions of length; $m$ and $n$ are positive integers.

The potential parameters representing the interaction between different type atoms can be written from the Lorentz-Berthelet as follows [22]:

$$
\begin{aligned}
& A_{i j}=A_{j i}=\frac{A_{i}+A_{j}}{2}, \quad n_{i j}=n_{j i}=\frac{n_{i}+n_{j}}{2}, \\
& m_{i j}=m_{j i}=\frac{m_{i}+m_{j}}{2}, \\
& \varepsilon_{i j}=\varepsilon_{j i}=\sqrt{\varepsilon_{i} \varepsilon_{j}} .
\end{aligned}
$$

TABLE

The Sutton-Chen EAM potential parameters for PdNi model alloy system [23].

\begin{tabular}{c|c|c|c|c}
\hline \hline Interaction & $A[\AA]$ & $\varepsilon[\mathrm{eV}]$ & $n$ & $m$ \\
\hline $\mathrm{Pd}-\mathrm{Pd}$ & 3.89 & $4.1260 \times 10^{-3}$ & 12 & 7 \\
$\mathrm{Ni}-\mathrm{Ni}$ & 3.52 & $1.5714 \times 10^{-2}$ & 9 & 6
\end{tabular}

The potential parameters for $\mathrm{Pd}-\mathrm{Pd}, \mathrm{Ni}-\mathrm{Ni}$, and $\mathrm{Pd}-\mathrm{Ni}$ atomic interactions have been given in Table as Ref. [23].

\subsection{The Honeycutt-Andersen (HA) method}

It is known that the pair analysis technique can be used to describe the microscopic local structure and short-range order in the structures [24]. In this technique the local structure is classified by using a sequence of four integers $(i j k l)$ which have the following meanings, the first integer $(i)$ is 1 when the atoms in the root pair are bonded, otherwise it is 2 . The second integer $(j)$ is the number of near-neighbour atoms shared in common by the root pair. The third integer $(k)$ is the number of nearest-neighbour bonds among the shared neighbours. The fourth integer $(l)$ is needed to difference between the cases when the first three indices are the same but the bond geometries are different.

It is believed that the method is convenient to analyze the geometric characteristics of an atomic cluster. If any atom system $\mathrm{A}-\mathrm{B}$ forms a bond, $i=1$, otherwise $i=2$, $\mathrm{A}$ and $\mathrm{B}$ atoms represent root pair. For example, the 1551 bonded pairs represent the two root pair atoms with five common neighbours that have five bonds forming a pentagon of near-neighbour contact. In this case, some typical bonded pairs are examined to understand the local structure of systems. The 1551 bonded pairs have fivefold symmetry, and the ratio of 1551 bonded pairs gives a measure of the degree of ideal icosahedral (ICOS) order. The 1421 and 1422 bonded pairs are characteristic bonded pairs for fcc and hcp crystal structures, respectively. The 1661 and 1441 bonded pairs are the characteristic bonded pairs for bcc crystal structure [24]. The pair fractions shown here are normalised so that the sum over all cases for the nearest neighbours is summed to unity. For example, a perfect fcc cluster having 13 atoms leads to 0.33 indicating 1421 bonded pairs, whereas perfect bulk fcc leads only to 11421 bonded pairs (namely $100 \% 1421$ bonded pairs). Perfect bcc cluster with 15 atoms has 0.121441 bonded pairs and 0.161661 bonded pairs, bulk bcc structure has 0.43 and 0.571441 and 1661 bonded pairs, respectively. The 1541 and 1431 bonded pairs are characteristic disordered ICOS order. The 1201 , 1331, 1321, 1311 bonded pairs represent the rhombus symmetrical features of short-range order.

\section{Simulation procedure}

The simulations were performed with the system consisting of 10976 atoms in a cubic box with periodic boundary conditions along all the three directions. The fcc structure for the $\mathrm{Pd}-55 \% \mathrm{Ni}$ model alloy systems has been chosen as the starting configuration for the MD computer simulation. The $6037 \mathrm{Ni}$ atoms were randomly assigned on the fcc lattice point. The potential functions were truncated at a cut-off distance of $2.2 A_{\mathrm{PdPd}}$. The temperature of the system has been controlled by rescaling the atomic velocities at every two integration steps. The equation of motion is numerically integrated by the Gears 5th order predictor-corrector algorithm. The molecular dynamics time step was set up as $8.034 \mathrm{fs}$.

The simulation has been started at $2000 \mathrm{~K}$ temperature value. After $50000 \mathrm{MD}$ steps when the model system has reached to stable liquid phase at $0 \mathrm{GPa}$, it is then cooled from 2000 to $300 \mathrm{~K}$ with two different cooling rates, $2 \times 10^{12}$ and $2 \times 10^{13} \mathrm{~K} / \mathrm{s}$. This process was also carried out for $5 \mathrm{GPa}$ pressure value.

\section{Result and discussion}

We analyze the radial distribution function (RDF) $g(r)$, to investigate the structural properties during the cooling process. The variation with temperatures of the RDF for PdNi alloy system at cooling rate of $2 \times 10^{12} \mathrm{~K} / \mathrm{s}$ 
at $0 \mathrm{GPa}$ and $5 \mathrm{GPa}$ are given in Fig. 1a and b. The model structure is in liquid phase for two pressure values at the $2000 \mathrm{~K}$ temperature. Cooling to $1000 \mathrm{~K}$, we still observe the structure of liquid, in fact a supercooled liquid, while the model alloy system is in an amorphous state during cooling process. A splitting can be seen in the second peak of the RDF at the $700 \mathrm{~K}$ (Fig. 1a) and $800 \mathrm{~K}$ (Fig. 1b) for 0 and $5 \mathrm{GPa}$ pressure values. This splitting is a characteristic of the metallic glass [25]. Then, as the temperature decreased the splitting becomes sharper. The RDF shows an amorphous structure for two pressure values at the cooling rate of $2 \times 10^{12} \mathrm{~K} / \mathrm{s}$ and $300 \mathrm{~K}$. The intensities of the first peak of the RDF curves increase with decreasing temperature, so it can be said that the nearest neighbour coordination is raised during solidification of PdNi alloy.

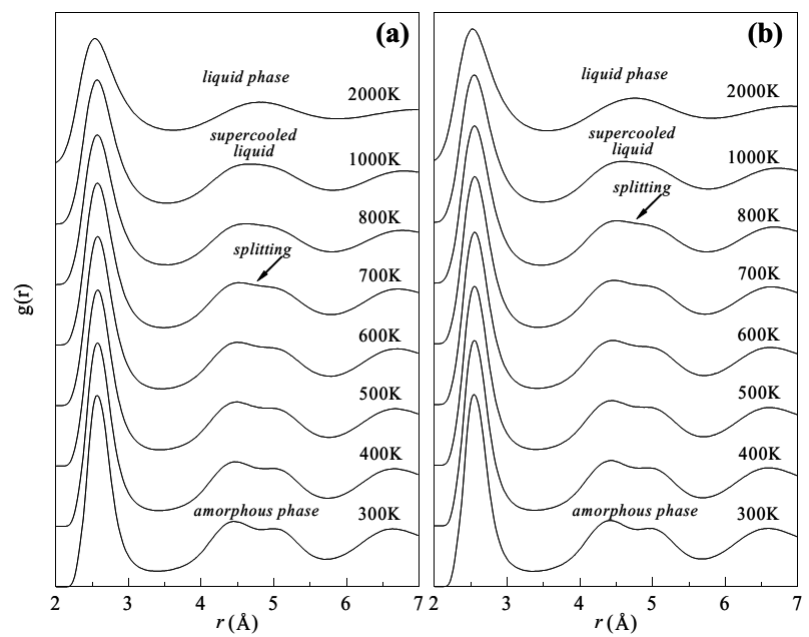

Fig. 1. Radial distribution function under pressure values (a) $0 \mathrm{GPa}$, (b) $5 \mathrm{GPa}$ at cooling rate of $2 \times 10^{12} \mathrm{~K} / \mathrm{s}$ for PdNi alloy system.

The temperature dependence of the Wendt-Abraham parameter is a sensitive measure of the glass transition. To define the glass transition temperature in MD simulations the Wendt-Abraham parameter defined by $g_{\min } / g_{\max }$ is used [26]. Here $g_{\min }$ is first minimum value and $g_{\max }$ is first maximum value of RDF curve.

The formation of a glass leads to a change in slope and intersection of two straight lines yields $T_{\mathrm{g}}$. Figure 2 gives the relationship between $g_{\min } / g_{\max }$ and temperature for two different cooling rates $\left(2 \times 10^{12}\right.$ and $\left.2 \times 10^{13}\right)$ and pressures $(0$ and $5 \mathrm{GPa})$. There exist two lines with different slopes for each cooling rate and pressures in Fig. 2. $T_{\mathrm{g}}$ is taken to be the temperature at which these lines intersect with each other, as shown in Fig. 3 plotted for cooling rate of $2 \times 10^{12} \mathrm{~K} / \mathrm{s}$ and $0 \mathrm{GPa}$.

It is determined from Fig. 2 that the glass forming temperature of PdNi binary alloy system for $2 \times 10^{12} \mathrm{~K} / \mathrm{s}$ cooling rate under the pressure values of 0 and $5 \mathrm{GPa}$ are 700 and 800 , respectively and for $2 \times 10^{13} \mathrm{~K} / \mathrm{s}$ cooling rate under same pressure values 820 and $900 \mathrm{~K}$, respectively.

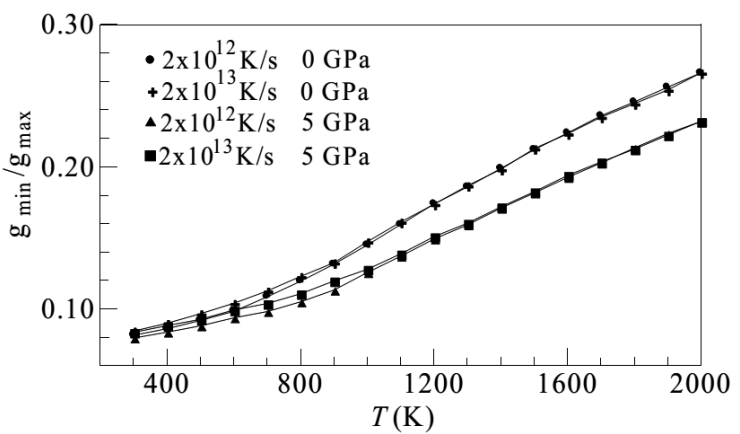

Fig. 2. The glass transition temperature at different cooling rates and under pressure values for $\mathrm{PdNi}$ alloy system.

As seen from the figure, the increase of the pressure and cooling rates increases the glass transition temperature.

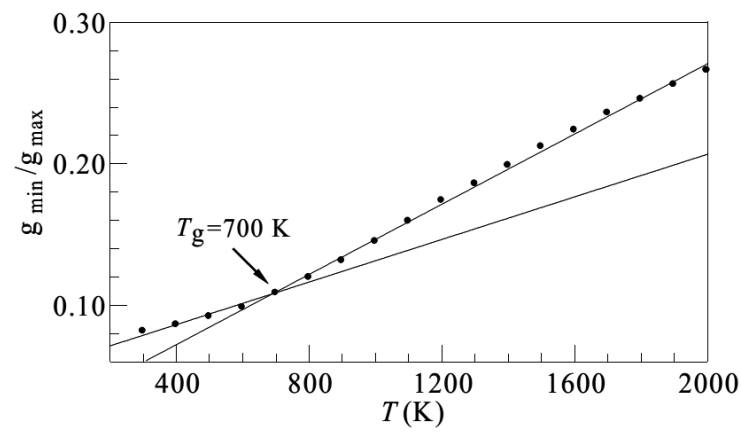

Fig. 3. $g_{\min } / g_{\max }$ is fitted to a linear function to estimate the glass temperature at the $2 \times 10^{12}$ cooling rate and at the 0 GPa pressure value.

From the study performed on $\mathrm{Pb}[7]$ and $\mathrm{Se} \mathrm{[10]} \mathrm{matter,}$ it was determined that the glass forming temperature was increased with applied pressure. Under high pressure, the liquid atoms compress each other causing a much higher density and a smaller amount of free volume. As a result, the decrease in the moving space for particles due to high pressure increases $T_{\mathrm{g}}$ value [10]. Also, the glassing forming temperature increases with increasing cooling rates. Slower cooling rates allow a greater amount of molecular relaxation to occur in the liquid phase before the structure is frozen at the $T_{\mathrm{g}}$, hence the glass forms at a lower temperature [6].

In order to analyze the local arrangements, the atomic bonded pairs information of the model alloy system was examined from the atomic bonding view using the HA pair analyses method. This method can be used to distinguish various local structures, especially, crystalline unit cells and liquids environments. Most of bonded pairs are of 1421 in fcc, 1422 and 1421 in hcp, 1441 and 1661 in bcc, 1551, 1431 and 1541 bonded pairs in liquid and glasses, as shown in Fig. 4. Specially, an ideal icosahedron consists of 12,1551 bonded pairs. 1541 and 1431 bonded pairs represent a type of defected icosahedral order. 

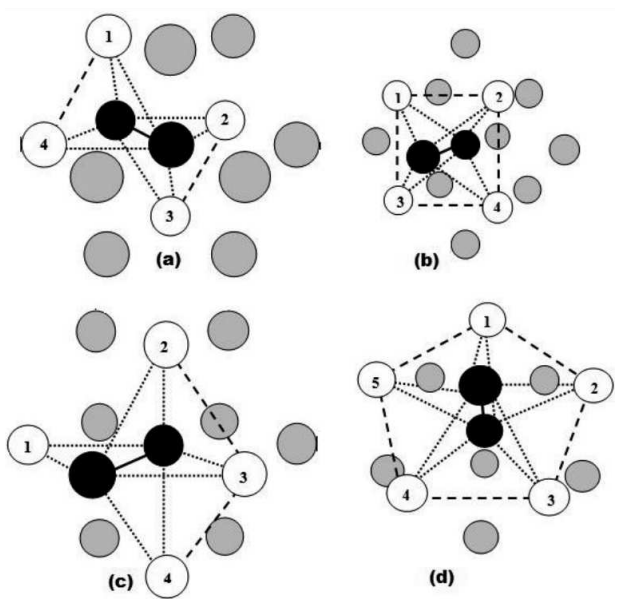

Fig. 4. Black atoms (A and B) represent pair atoms (root pair); white atoms represent neighbour atoms with root pair. Straight thick line denotes the bond between $\mathrm{A}$ and $\mathrm{B}$, dotted lines denote near-neighbour bond numbers of root pair, dashed lines denote bond among neighbours. (a) 1421 bonded pair in fcc cluster, (b) 1441 bonded pair in bcc cluster, (c) 1422 bonded pair in hcp cluster, (d) 1551 bonded pair in icos cluster.
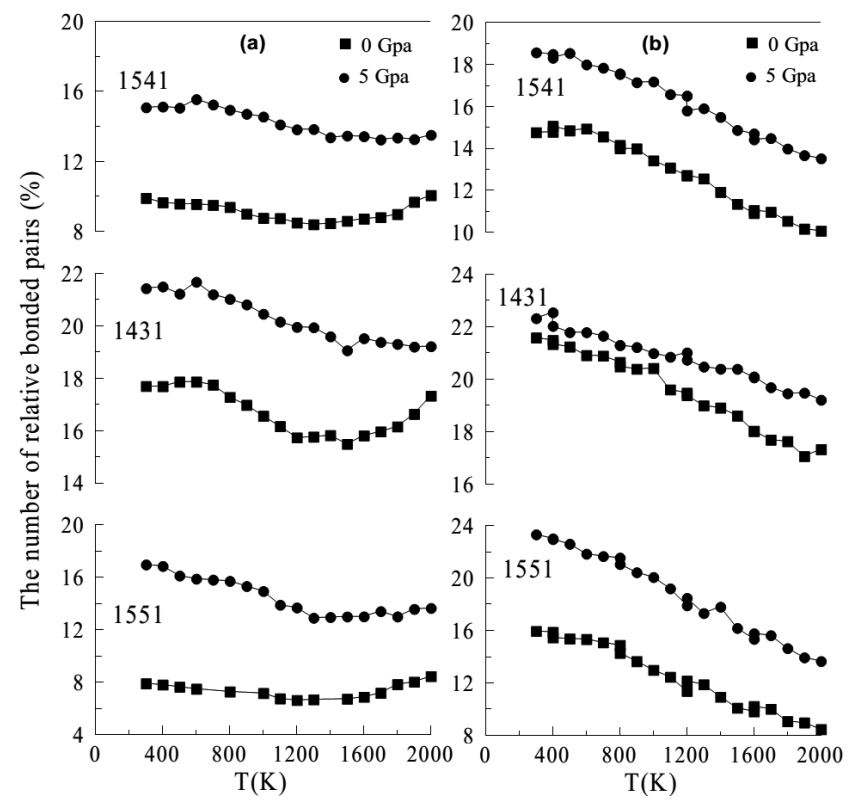

Fig. 5. Relative number of various bonded pairs with the temperature. (a) $2 \times 10^{12} \mathrm{~K} / \mathrm{s}$ cooling rate, (b) $2 \times 10^{13} \mathrm{~K} / \mathrm{s}$ cooling rate.

Figure 5 shows the relevant numbers of various amorphous-type bonded pairs under different pressures and cooling rates. The results say that the number of 1551, 1541 and 1431 bonded pairs increases rapidly as the temperature drops. The amount of 1551, 1541 and 1431 bonded pairs is much more than other bonded pairs in amorphous (supercooled) phase which suggests that these bonded pairs are main structural units in amor- phous phase. The total number of bonded pairs (1551, 1541 and 1431) increase with the increasing cooling rate. On the other hand, the amount of 1551, 1541 and 1431 bonded pairs at high pressure is bigger than that at normal pressure for two cooling rates. These results demonstrated that the icosahedral and defective icosahedral ordering degree is increased in the model alloy system with the increasing cooling rate at both normal and high pressure.
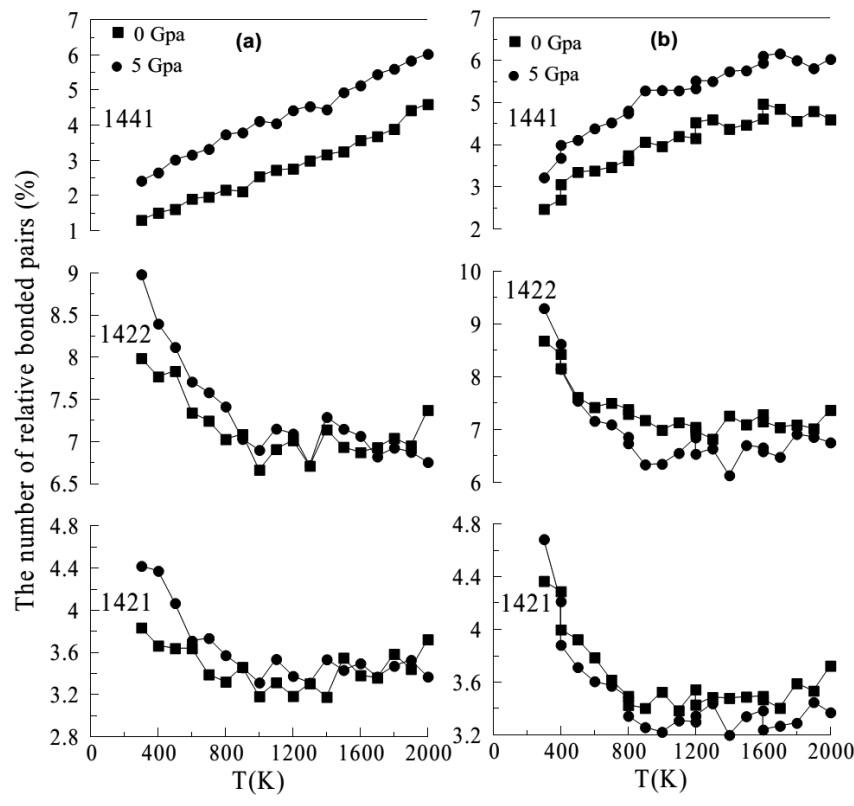

Fig. 6. Relative number of various bonded pairs with the temperature. (a) $2 \times 10^{12} \mathrm{~K} / \mathrm{s}$ cooling rate, (b) $2 \times 10^{13} \mathrm{~K} / \mathrm{s}$ cooling rate.

The presence of 1421, 1422 and 1441 bonded pairs indicates that crystalline type bonded pairs can exist in amorphous phase, but the number of these bonded pairs is a very small proportion as shown in Fig. 6. Especially, as the number of fcc and hcp type bonded pairs increase, the number of bcc type bonded pairs decrease with decreasing temperature of both cooling rates for model alloy. This status explain that some bcc unit cell does not exist in a proportion comparable to icosahedral order and defective icosahedral at low temperatures. As the temperature falls down, because of packing defects and frustration effect, amorphous phase does not contain only ideal icosahedral type bonded pairs, at the same time these distortions required for other amorphous type bonded pairs, such as 1431 and 1541 defect icosahedral type bonded pairs. These results agree with previous studies $[8,27]$. It should be pointed out that high pressure favors the 1421, 1422 and 1441 bonded pairs during cooling process at two cooling rates.

\section{Conclusion}

In this study, we have performed molecular dynamics simulation to study the glass structure properties of 
PdNi alloy during the different cooling rates under 0 and $5 \mathrm{GPa}$ pressures. The interaction potential between atoms is represented by SCEAM. For two applied pressures value shows amorphous structures at the cooling rate of $2 \times 10^{12}$ and $2 \times 10^{13} \mathrm{~K} / \mathrm{s}$, respectively. It can be seen clearly that liquid-amorphous transition temperatures are strongly dependent on the applied pressure. The glass forming temperature $T_{\mathrm{g}}$ increases with increasing both cooling rate and external pressure values. High pressure strengthens the order degree of the glass $\mathrm{PdNi}$ model alloy system and favours the glass formation. As the temperature drops, the number of $1551,1541,1431$, 1421 and 1422 bonded pairs increase, on the contrary, the number of 1441 bonded pairs is decreased for model alloy system. Especially, the number of the ideal icosahedra under high pressure contributes to the glass formation of the $\mathrm{Pd}-\mathrm{Ni}$ model alloy system.

\section{References}

[1] R.D.S. Lisboa, C.S. Kiminami, J. Non-Cryst. Solids 304, 36 (2002).

[2] Z.X. Wang, R.J. Wang, W.H. Wang, Mater. Lett. 60, 831 (2005).

[3] L. Hui, B. Xiufang, Z. Jingxiang, Mater. Sci. Eng. A 271, 116 (1999).

[4] H.R. Cong, X.F. Bian, J.X. Zhang, H. Li, Mater. Sci. Eng. A 326, 343 (2002).

[5] L. Qi, H.F. Zhang, Z.Q. Hu, P.K. Liaw, Phys. Lett. A 327, 506 (2004).

[6] S. Özdemir Kart, M. Tomak, M. Uludoğan, T. Çagın, Mater. Sci. Eng. A 435-436, 737 (2006).

[7] J. Liu, J.Z. Zhao, Z.Q. Hu, Comput. Mater. Sci. 37, 234 (2006).

[8] L. Hui, F. Pederiva, Chem. Phys. 304, 261 (2004).

[9] Z.X. Wang, R.J. Wang, W.H. Wang, Mater. Lett. 60, $831(2005)$.
[10] F. Shimojo, K. Hoshino, Y. Zempo, J. Non-Cryst. Solids 312-314, 388 (2002).

[11] T. Çă̆ın, G. Dereli, M. Uludoğan, M. Tomak, Phys. Rev. B 59, 3468 (1999).

[12] R.A. Johnson, Phys. Rev. B 39, 12554 (1989).

[13] L.A. Marqués, L. Pelaz, M. Aboy, P. Lopez, J. Barbolla, Comput. Mater. Sci. 33, 92 (2005).

[14] Y. Shao, P.C. Clapp, J.A. Rifkin, Metall. Mater. Trans. A 27, 1477 (1996).

[15] M.S. Daw, R.D. Hatcher, Solid State Commun. 56 , 697 (1985).

[16] A.F. Voter, S.P. Chen, Mater. Res. Soc. Symp. Proc. 82, 175 (1987).

[17] M.W. Finnis, J.E. Sinclair, Philos. Mag. 50, 45 (1984).

[18] A.P. Sutton, J. Chen, Philos. Mag. Lett. 61, 139 (1990).

[19] M. Grujicic, P. Dang, Mater. Sci. Eng. A 201, 194 (1995).

[20] J. Gui, Y. Cui, S. Xu, Q. Wang, Y. Ye, M. Xiang, R. Wang, J. Phys., Condens. Matter 6, 4601 (1994).

[21] D. Caprion, H.R. Schober, J. Non-Cryst. Solids 326, 369 (2003).

[22] Y. Qi, T. Çağnn, Y. Kimura, W.A. Goddard, Phys. Rev. B 59, 3527 (1999).

[23] H. Rafii-Tabar, A.P. Sutton, Philos. Mag. Lett. 63, 217 (1991).

[24] J.D. Honeycutt, H.C. Andersen, J. Phys. Chem. 91, 4950 (1987).

[25] D. Caprion, H.R. Schober, J. Non-Cryst. Solids 326327, 369 (2003).

[26] L. Qi, H.F. Zhang, Z.Q. Hu, Intermetallics 12, 1191 (2004).

[27] L. Qi, L.F. Dong, S.L. Zhang, Z.Q. Cui, M.Z. Ma, Q. Jing, G. Li, R.P. Liu, Comput. Mater. Sci. 42, 713 (2007). 